\title{
Construção de instrumento de avaliação do pensamento crítico: considerações
}

\author{
preliminares
}

\section{Construction of an instrument for the evaluation of critical thinking: previous \\ considerations.}

\author{
Bruna Casiraghi*, Leandro S. Almeida *, Júlio C. S. Aragão* \& Luciana P. Brito** \\ *Universidade do Minho, **Instituto Politécnico de Viana do Castelo.
}

\begin{abstract}
Resumo
Existem diversos instrumentos validados para a avaliação do pensamento crítico, contudo os instrumentos são amplos, longos e indisponíveis para aplicação em larga escala, pelo menos em língua portuguesa. Este trabalho objetiva apresentar o primeiro momento de elaboração de um instrumento de avaliação do pensamento crítico, com alguma facilidade de aplicação e que abarque as habilidades essenciais, para estudantes do ensino superior. Até ao momento este processo compreendeu: levantamento e análise da bibliografia; identificação dos processos cognitivos essenciais; definição do formato do problema e respostas a considerar; discussão com especialistas; revisão e adequação; aplicação e análise qualitativa das situações junto dos respondentes. Até ao momento, a principal conclusão é que este processo é moroso e difícil, sendo certo que a relevância da avaliação do pensamento crítico na população universitária justifica a prossecução do projeto.

Palavras-chave: Pensamento crítico, Ensino superior, competências transversais, avaliação
\end{abstract}

\begin{abstract}
There are several validated instruments for the evaluation of critical thinking, however the instruments are broad, long and unavailable for large scale application, at least in Portuguese. This paper aims to present the first moment of elaboration of an instrument of evaluation of critical thinking, easy to apply and that includes the essential skills enabling the accompaniment in higher education. So far this process has included: survey and analysis of the bibliography; identification of essential cognitive processes; definition of the problem format and answers to consider; discussion with experts; review and adequacy; application and qualitative analysis of the situations with the respondents. So far, the main conclusion is that this process is time consuming and difficult, since the relevance of critical thinking evaluation in university population justifies the continuation of the project.

Keywords: Critical thinking, higher education, transversal skills, evaluation
\end{abstract}

\section{Introdução}

O pensamento crítico (PC) é entendido como um raciocínio de complexidade superior, que articula os conhecimentos com as habilidades cognitivas voltadas para a tomada de decisões que levam a resultados mais satisfatórios (Amorim, 2013; Dias, Franco, Almeida, \& Joly, 2011). De acordo com Halpern (2002), pensamento crítico pode ser definido como o uso das habilidades cognitiva ou estratégias que aumentam a probabilidade de um resultado desejável e tem como característica ser intencional, fundamentado e orientado para os objetivo. Franco e Almeida (2017) enfatizam a importância do pensamento crítico em diferentes âmbitos da vida no cotidiano, principalmente no desempenho académico ou profissional, nas quais as competências transversais como o PC são cada vez mais valorizadas.

Tais competências configuram-se como os principais objetivos educacionais, principalmente no ensino superior, considerando a necessidade de formar profissionais capacitados a atuarem na realidade, acompanhando as constantes atualizações e mudanças no conhecimento disponível, possibilitando tomadas de decisão e resolução de problemas de forma efetiva (Almeida \& Franco, 2011; Amorim, 2013; Butler et al., 2012; Esteves, 2008; Pereira \& Alich, 2015; Saiz \& Rivas, 2008; Zimmerman, 2002).

$\mathrm{Na}$ medida em que o Ensino Superior deve garantir o desenvolvimento do PC, instrumentos capazes de avaliar a capacidade de pensar criticamente, assim como o seu desenvolvimento durante a formação, tornam-se fundamentais (Ennis, 1993; Ku, 2009). Considerando tal realidade e a necessidade de testes que atendam a esse objetivo em língua portuguesa, este trabalho pretende apresentar a etapa da definição e elaboração da questão problema-tipo a considerar no desenvolvimento de um instrumento de avaliação do PC voltado ao ensino superior que se preste ao mapeamento geral de instituições ou populações, garantindo os atributos de validade, especificidade e confiabilidade.

O projeto é desenvolvido por uma equipe de trabalho e foi elaborado a partir dos seguintes processos: levantamento e análise da bibliografia, que permitiu a definição dos processos fundamentais; elaboração de questão problema; discussão com especialistas; revisão e adequação das questões; aplicação a pequenos grupos de alunos e análise dos seus comentários e resultados. 


\section{Desenvolvimento}

A análise da bibliografia pertinente a avaliação do PC permite mapear as ações na área, indicando os pontos relevantes, os processos facilitadores e as principais dificuldades. Dentre os testes voltados ao perfil do aluno do Ensino Superior, os instrumentos mais referenciados e utilizados internacionalmente (Franco \& Almeida, 2017) são: Watson-Glaser Critical Thinking Appraisal (CTAI) (Watson \& Glaser, 1980); Ennis-Weir Critical Thinking Essay Test (Ennis-Weir) (Ennis \& Weir, 1985); Cornell Critical Thinking Test (CCTST) (Ennis, Millman, \& Tomko, 1985); California Critical Thinking Skills Test (Facione, 1990); Halpern Critical Thinking Assessment Using Everyday Situations (Halpern, 2007) e PENCRISAL - Pensamento, crítico, Salamanca (Rivas \& Saiz, 2012).

Dos instrumentos citados, três deles são constituídos somente com questões de múltipla escolha (CTAI, California, CCTST), dois com questões abertas (Ennis-Weir e PENCRISAL) e o HCTA possui tanto questões de múltipla escolha como questões abertas. As questões de múltipla escolha possibilitam maior facilidade na correção e agilidade na execução das provas, no entanto, alguns autores defendem que não é possível avaliar toda a complexidade do PC através deste modelo de questão (Saiz \& Rivas, 2008), considerando a necessidade de explicitar os processos cognitivos complexos envolvidos.

A análise da bibliografia permitiu estabelecer as principais dimensões do PC avaliadas nos testes, que podem ser agrupadas em três etapas principais: análise de argumento; explicação ou fundamentação de princípios ou dados; e tomada de decisão/resolução de problemas. $\mathrm{Na}$ análise de argumentos estão contemplados processos como avaliar argumentos, reconhecer pressupostos, incorporar o ponto de vista, análise da semântica, interpretação, raciocínio verbal entre outros. Dos testes citados, somente o PENCRISAL não avalia esta primeira etapa do PC. A explicação e fundamentação, que diz respeito a compreensão do problema, a busca de hipóteses explicativas e de solução, estão contemplados em todos os testes e refere-se ao raciocínio inferencial, dedutivo e indutivo, a elaboração de hipóteses e premissas, explicação, avaliação, probabilidade e incerteza etc. A última etapa e mais difícil de ser avaliada, já que depende não só de aspectos cognitivos, mas também motivacionais, é a tomada de decisão e resolução de problemas, que só são avaliados nos testes HCTA e PENCRISAL.

Considerando o formato dos testes existentes, optou-se pela elaboração de um instrumento misto, constituído de questões abertas e de múltipla escolha, que possa ser realizado no tempo máximo de 40 minutos e com a correção a ser feita em no máximo 10 minutos, baseado em problemas do cotidiano dos estudantes, sem que seja necessário o domínio de conhecimentos específicos para sua solução.

A elaboração da questão problema partiu do levantamento de problemas comuns entre estudantes do ensino superior para a definição de um assunto a ser abordado. Uma questão modelo foi formulada e submetida a uma sessão de discussão com especialistas na área, com experiência em elaboração de testes de PC e programas de desenvolvimento. Os especialistas indicaram a necessidade de adequações no formato e no conteúdo. A situação problema foi inteira reformulada e resubmetida aos especialistas, em nova reunião técnica, na qual outras questões foram apontadas e outras situações problema foram amplamente discutidas.

Após novas reformulações, a situação problema foi testada e discutida com alunos de mestrado e do doutorado para avaliar a clareza, a dificuldade e o potencial em eliciar o PC. As respostas obtidas indicaram que o enunciado do problema estava claro, os alunos relataram uma experiência positiva ao executar a tarefa, contudo, como todos os alunos atingiram o objetivo, ficou evidente a necessidade de novas adequações por forma a aumentar a sensibilidade do instrumento.

\section{Considerações finais}

A elaboração e validação de um instrumento psicométrico é uma tarefa que envolve amplo estudo e várias etapas de desenvolvimento e testagem, como recomendam os especialistas e organismos internacionais. A explicitação de tais etapas e dificuldades encontradas permite compreender os processos inerentes, resultados alcançados e o tipo de instrumento que vai ser colocado à disposição da comunidade, assim como pode orientar novos projetos com objetivo semelhante.

Um instrumento de avaliação do PC, embasado na bibliografia sobre o assunto e nos testes já existentes, para que seja confiável, deve conter questões que possibilitem identificar funções cognitivas complexas. Além disso, a situação problema deve envolver conflitos vivenciados pelos estudantes universitários em seu cotidiano, que ofereça os dados necessários para se fazer as deduções e inferências, assim como selecionar dados relevantes e irrelevantes, e possibilite uma tomada de decisão que seja resultado do pensamento crítico. Ao mesmo tempo, a situação deve manter um nível de complexidade que exija reflexão e raciocínio para sua solução. Obter este equilíbrio é uma tarefa complexa e dinâmica que exije dos formuladores criatividade e conhecimento das dinâmicas envolvidas na operação do PC.

Embora a tarefa esteja longe de se considerar concluída, o processo aqui descrito permitiu à equipe um melhor delineamento das etapas de produção necessárias para o desenvolvimento e validação do instrumento proposto. Três ideias são, então, centrais na prova a construir: (i) escolha dos processos cognitivos essenciais à avaliação do PC sem a necessidade de abarcar todos os processos constantes de provas similares; (ii) um formato de resposta que possibilite aos sujeitos escolher e justificar respostas, evitando situações de "itens de múltipla escolha" com base em alternativas de resposta facultadas ou redação de respostas abertas que dificultam a sua análise e cotação posterior; e (iii) uma prova cuja extensão não seja demasiada, tornando possível uma avaliação em torno dos 30-40 minutos. 


\section{Referêcias}

Almeida, L. S., \& Franco, A. H. R. (2011). Critical thinking: its relevance for education in a shifting society. Revista de Psicología, 29(1), 175-195.

Amorim, M. M. P. (2013). Pensamento crítico nos estudantes e profissionais da área de saúde. Universidade Fernando Pessoa, Porto.

Butler, H. A., Dwyer, C. P., Hogan, M. J., Franco, A., Rivas, S. F., Saiz, C., \& Almeida, L. S. (2012). The Halpern Critical Thinking Assessment and real-world outcomes: Cross-national applications. Thinking Skills and Creativity, 7(2), 112-121. https://doi.org/10.1016/j.tsc.2012.04.001

Dias, A. S., Franco, A. H. R., Almeida, L. S., \& Joly, C. (2011). Competências de estudo e pensamento crítico em alunos universitários. Em XI Congreso Internacional Galego-Portugués de Psicopedagoxía (pp. 4647-4654). Universidade da Coruña. Obtido de http://repositorium.sdum.uminho.pt/handle/1822/1566 2

Ennis, R. H. (1993). Critical thinking assessment. Theory Into Practice, 32(3), 179-186. https://doi.org/10.1080/00405849309543594

Esteves, M. (2008). Para a excelência pedagógica do ensino superior. Sísifo: Revista de Ciências da Educação, (7), 101-110.

Franco, A., \& Almeida, L. (2017). Definição e medida do pensamento crítico. Em Criatividade e pensamento crítico: conceito, avaliação e desenvolvimento (pp. 107-132). Porto: CERPSI/ Centro de Estudos e Recursos em Psicologia.

Halpern, D. F. (2002). Thought and Knowledge: An Introduction to Critical Thinking, 4th Edition (5th ed.). London: Lawrence Erlbaum Associates.

Ku, K. Y. L. (2009). Assessing students' critical thinking performance: Urging for measurements using multi-response format. Thinking Skills and Creativity, 4(1), 70-76. https://doi.org/10.1016/j.tsc.2009.02.001

Pereira, S., \& Alich, V. (2015). A avaliação do pensamento crítico numa perspetiva psicológica. Em Dominguez, Caroline et al. (Ed.), Pensamento crítico na educação. Desafios atuais (pp. 87-98). Vila Real: Universidade de Tras-os-Montes e Alto Douro, UTAD.

Rivas, S. F., \& Saiz, C. (2012). Validación y propiedades psicométricas de la prueba de pensamiento crítico PENCRISAL. Revista Electrónica de Metodologia Aplicada (Eletronic Journal of Apllied Methodology), 17(1), 18-34.

Saiz, C., \& Rivas, S. F. (2008). Evaluación del pensamiento crítico: una propuesta para diferenciar formas de pensar. ergo. Obtido de https://www.researchgate.net/publication/254833430_ Evaluacion_del_pensamiento_critico

Zimmerman, B. J. (2002). Becoming a Self-Regulated Learner: An Overview. Theory Into Practice, 41(2), 64-70. https://doi.org/10.1207/s15430421tip4102_2 